\title{
ESTRATÉGIAS NA ELABORACÃO DE PLANOS DE APOIO PARA ALUNOS COM DESENVOLVIMENTO ATÍPICO
}

\author{
Nathalie Baril \\ Universidade Federal do Paraná \\ natibaril@hotmail.com \\ Maria de Fátima Minetto \\ Universidade Federal do Paraná \\ fa.minetto@gmail.com \\ lasmin Zanchi Boueri \\ Universidade Federal do Paraná \\ boueri.iasmin@gmail.com \\ Sabrina Fernandes de Castro \\ Universidade Federal de Santa Maria \\ sabrinafcastro@gmail.com \\ Thais Carolina Albach Carniel \\ Universidade Federal do Paraná \\ thaiscarolinaufpr@gmail.com
}

Recepción Artículo: 17 mayo 2021

Admisión Evaluación: 17 mayo 2021

Informe Evaluador 1: 22 mayo 2021

Informe Evaluador 2: 27 mayo 2021

Aprobación Publicación: 02 junio 2021

\section{RESUMO}

Os planejamentos individualizados assumem nomenclaturas diversas e funções com diferentes abrangências, como é o caso do Plano de Apoio Centrado no Aluno com desenvolvimento atípico. 0 objetivo geral do presente estudo foi desenvolver estratégias para a elaboração de planos de apoio centrados em alunos com desenvolvimento atípico. A partir deste, foram estabelecidos objetivos específicos: descrever as prioridades elencadas pela equipe de planejamento e elaborar um plano de apoio centrado no aluno com desenvolvimento atípico. Para atingir os objetivos propostos, foi necessário consultar os resultados da aplicação de dois instrumentos utilizados em uma pesquisa prévia com os mesmos participantes: os questionários sociodemográficos e a Escala de Intensidade de Apoio (SIS-C). 0 instrumento utilizado nesta pesquisa foi 0 Guia para a Elaboração do Plano Centrado no Aluno, elaborado por uma das autoras. Os participantes foram quatro mães e quatro professoras de alunos com desenvolvimento atípico, sendo que dois frequentavam uma escola regular e os outros dois uma escola de educação básica na modalidade de educação especial. Também participaram de uma das etapas da pesquisa profissionais da escola de cada aluno, incluindo terapeutas e pedagogos, configurando-se em uma pesquisa de caráter predominantemente qualitativo. Os resultados mostraram que, com as estratégias propostas, foi possível elaborar um plano para cada aluno. Conclui-se que todo o processo de construção deste plano é a maior contribuição desta pesquisa para futuros profissionais que pretendam dar continuidade em pesquisas que envolvam planos de apoios individuais.

Palavras chave: planos individuais; escala de intensidade de apoio; comportamento adaptativo

\section{ABSTRACT}

Strategies in the preparation of support plans for students with atypical development. Individualized planning takes on different nomenclatures and functions with different scopes, such as the Support Plan Centered on Students with atypical development. The general objective of this study was to develop strate- 


\section{ESTRATÉGIAS NA ELABORAÇÃo dE PLANOS DE APOIO PARA ALUNOS COM DESENVOLVIMENTO ATÍPICO}

gies for preparing support plans centered on students with atypical development. Specific objectives were then established: to describe the priorities listed by the planning team and to prepare a support plan centered on students with atypical development. To achieve the proposed objectives, it was necessary to consult the results of two instruments used in a previous survey and administered with the same participants, namely the sociodemographic questionnaires and the Supports Intensity Scale - Children's Version (SIS-C). The instrument used in this study was the Guide for Preparing the Student Centered Plan, developed by the author of this study. The participants were four mothers and four teachers of students with atypical development, two of whom attended a regular school and the other two attended an elementary education school with special education facilities. Education professionals from each student's school, including therapists and pedagogues, also participated in one of the stages of the study, so that this study was predominantly qualitative. The results showed that, using the strategies proposed, it was possible to develop a plan for each student. The conclusion is reached that the entire process of building this plan is the main contribution of this research to future professionals who intend to continue research involving individual support plans.

Keywords: individual plans; support intensity scale; adaptive behavior

\section{INTRODUÇÃO}

As políticas que envolvem a escolarização do Público-Alvo da Educação Especial (PAEE), composto por pessoas com deficiência, transtorno global do desenvolvimento ${ }^{1}$ e altas habilidades ou superdotação (Brasil, 2011), são temas em constante discussão. No Brasil, as legislações referentes à política da educação inclusiva enfatizam a universalização do PAEE na rede regular de ensino, conforme o Plano Nacional de Educação (2010) e a Lei Brasileira de Inclusão - LBI (2015).

Diferentemente dos outros estados do Brasil, em que não é possível que alunos com deficiência estejam matriculados apenas em escolas especializadas, no Estado do Paraná a Deliberação nํㅜ 02/2016 assegura que 0 Sistema Estadual de Ensino ofertará a Educação Especial preferencialmente na rede regular de ensino, sendo possível a matrícula do PAEE em escolas na modalidade de educação especial (Paraná, 2016). A compreensão desse contexto se mostra necessária diante da caracterização dos participantes do presente estudo, sendo alunos de escolas regulares e de escolas de educação básica na modalidade de educação especial.

A solidificação de políticas educacionais que reafirmam o compromisso com a diversidade humana nos diversos ambientes educacionais tira o foco da incapacidade sobre 0 aluno e 0 direciona para estratégias dinâmicas, planejamentos individualizados e apoios efetivos na escolarização (Mello \& Hostins, 2018; Thompson \& Viriyangka, 2014).

Tannus-Valadão e Mendes (2018) traçaram um panorama histórico acerca do conceito do Plano Educacional Individualizado (PEI), compreendido como um instrumento que auxilia o currículo oficial, especificando e estruturando o tipo de atividade e apontando qual apoio profissional é conveniente para cada aluno, de modo que haja estímulo no processo de ensino-aprendizagem.

Pletsch e Glat (2012) realizaram um estudo no qual elaboram o Plano de Desenvolvimento Educacional Individualizado PDEl nos moldes dos planos individuais de inclusão utilizados nas redes escolares de países da Europa e nos Estados Unidos. Esses referenciais sugerem que, a partir do PDEl, é possível promover estratégias pedagógicas individualizadas em três dimensões: no processo de aprendizagem escolar, nas habilidades sociais e nas habilidades necessárias para a inclusão laboral (Pletsch et al., 2010). 0 estudo corrobora com os resultados da pesquisa de Tannus-Valadão e Mendes (2018) quanto à importância do planejamento de estratégias pedagógicas, tendo como base a aplicação de um Plano Individualizado (PI) para favorecer o processo de aprendizagem, fortalecer a autonomia e melhorar a qualidade de vida dos alunos com desenvolvimento atípico.

Os planos de apoio individualizados são obrigatórios em vários países, como na Inglaterra, Holanda, Bélgica, Canadá e vários Estados dos Estados Unidos (Felce, 2004; Herps, Buntinx \& Curfs, 2013; Robertson et al., 2017). Para Herps et al. (2016), os planos de apoios individuais referem-se ao processo de desenvolvimento de metas, seleção de recursos e estratégias de apoio para o desenvolvimento de pessoas com deficiência intelectual. 
Para a AAIDD (American Association on Intellectual and Developmental Disability), o Planejamento Centrado na Pessoa deve ser desenvolvido por uma equipe de planejamento para identificar e organizar apoios personalizados (Thompson et al., 2017). Mas, para compreender e mensurar as necessidades de apoio para cada indivíduo, desenvolveu-se em 2004 a Escala de Intensidade de Apoio, em inglês Supports Intensity Scale - SIS² (Thompson et al., 2016).

A partir dos estudos nacionais e internacionais (Pletsch \& Glat, 2012; Tannus-Valadão \& Mendes, 2018; Felce, 2004; Herps, Buntinx \& Curfs, 2013; Robertson et al., 2017) sobre planos centrados na pessoa e planos de apoio, o presente estudo utilizará o termo: Plano de Apoio Centrado no Aluno (PACA). Associou-se o termo utilizado pela AAIDD (Thompson et al., 2017) no Guia para o planejamento centrado na pessoa com a SIS-A, e também estudos que utilizam o Plano de Apoio individual (Herps et al, 2016; Van Loon, 2015).

Para contribuir na qualidade de vida e na participação efetiva de cada aluno, foi traçado o objetivo geral do presente estudo: desenvolver estratégias para a elaboração de Planos de Apoio Centrados em Alunos com desenvolvimento atípico. Para complementar foram estabelecidos objetivos específicos: descrever as prioridades elencadas pela equipe de planejamento para elaboração do PACA e elaborar o PACA. Vale destacar que, para atingir os objetivos propostos, utilizou-se os resultados da SIS-C e dados sociodemográficos de uma pesquisa que antecedeu o presente estudo com os mesmos participantes (Baril, 2019).

\section{MÉTODO}

A presente pesquisa tem um caráter predominantemente qualitativo, do tipo transversal com desenho não experimental de caráter descritivo. Uma das pesquisadora foi parte integrante do diálogo entre escola e família, tornando o estudo com características de uma pesquisa colaborativa em uma de suas etapas.

Participaram desta pesquisa quatro mães e quatro professoras de alunos com desenvolvimento atípico. Em uma das etapas da pesquisa também participaram outros profissionais da escola especial, sendo uma psicóloga, uma terapeuta ocupacional e uma pedagoga.

A Figura 1 ilustra as principais informações referentes a cada aluno, sendo que cada um deles recebeu um nome fictício que remete a personagens de desenhos infantis ${ }^{3}$.

Figura 1 - Dados dos(as) alunos(as)

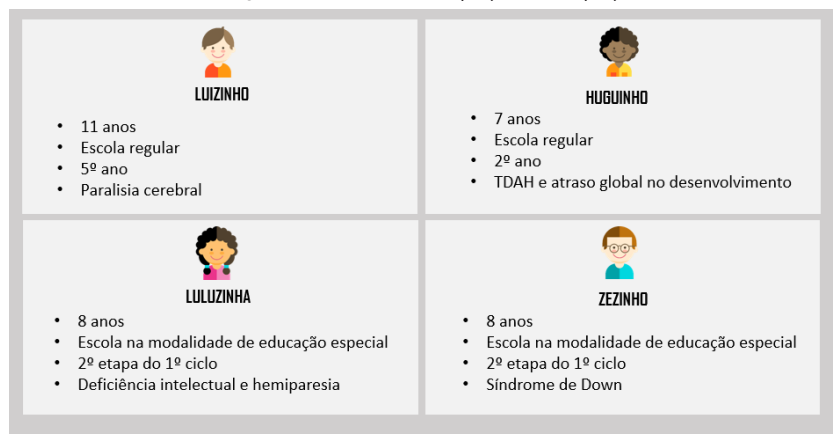

Fonte: $A$ autora (2019)

Todos os alunos frequentavam o Ensino Fundamental (EF). Luluzinha e Zezinho, alunos de uma escola na modalidade de educação especial, estão inseridos em um ciclo contínuo do ensino fundamental, sendo a $2^{0}$ etapa do $1^{0}$ ciclo correspondente ao $1^{0}$ no do EF.

As informações sociodemográficas a respeito das famílias e professoras podem ser visualizadas na Figura 2. 


\section{ESTRATÉGIAS NA ELABORAÇÃo DE PLANOS DE APOIO PARA ALUNOS COM DESENVOLVIMENTO ATÍPICO}

Figura 2 - Dados sociodemográficos referentes às famílias e professoras

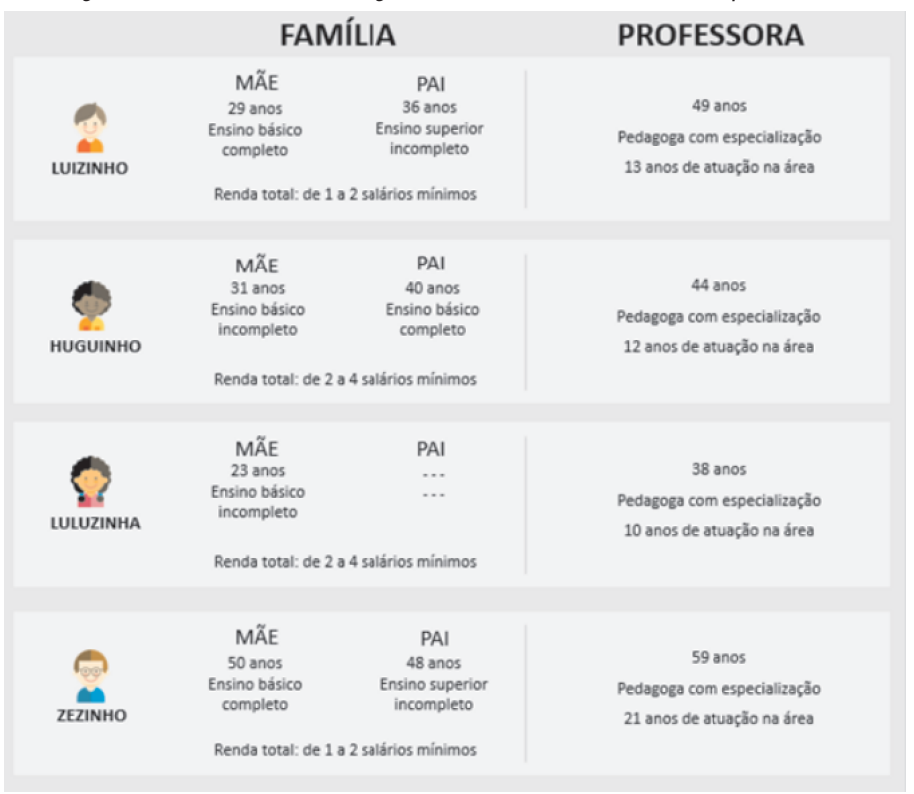

Fonte: A autora (2019)

Para a realização da presente pesquisa, foram utilizados os resultados de três instrumentos:

1- Questionários sociodemográficos; 2- Escala de Intensidade de Apoio para Crianças: SIS-C; 3- Guia para o desenvolvimento do plano de apoio centrado no aluno.

0 Questionário sociodemográfico é um material desenvolvido pela pesquisadora, orientadora e colaboradores, com base na entrevista e formulário do perfil, presentes na SIS-C (Thompson et al., 2016) e com perguntas adicionais para compor o perfil da amostra, como tempo de experiência profissional das professoras e renda salarial das famílias.

A SIS-C (Thompson et al., 2016), é um instrumento que mede a intensidade relativa das necessidades de apoio para crianças e adolescentes com idade entre 5 e 16 anos, com diagnóstico de deficiência intelectual e transtornos no neurodesenvolvimento. Avalia a intensidade específica de apoio necessário às crianças em ambientes típicos de acordo com a sua idade por meio das dimensões: frequência, tempo, e tipo, ao longo das 62 habilidades adaptativas divididas nas seguintes áreas: Parte A - Atividades de Vida diária; Parte B - Atividades na Comunidade; Parte C - Atividades de Participação Escolar; Parte D - Atividades de Aprendizagem Escolar; Parte E - Atividades de Saúde e Segurança; Parte F - Atividades Sociais; e Parte G - Atividades de Autoadvocacia. Além de avaliar as necessidades médico (19 itens) e comportamental (14 itens).

0 Guia para a elaboração do PACA foi um material instrumental elaborado pelas autoras, com base no Guia para Planejamento Centrado na Pessoa com a Escala de Intensidade de Apoio para Adultos (Thompson et al., 2017) e também no Manual do Usuário da Escala de Intensidade de Apoio para Crianças (Thompson et al., 2016).

Para o desenvolvimento do PACA foi necessário utilizar os resultados da SIC-C (Thompson et al., 2016), respondido pela mãe e à professora de cada aluno em momentos distintos. Esses resultados mostraram a intensidade de apoio necessária que cada aluno necessitava para realizar as atividades adaptativas citadas na escala de acordo com a percepção da mãe e da professora. Com esses resultados, seguiu-se as etapas descritas na Figura 3. 
Figura 3 - Passos para a elaboração do PACA

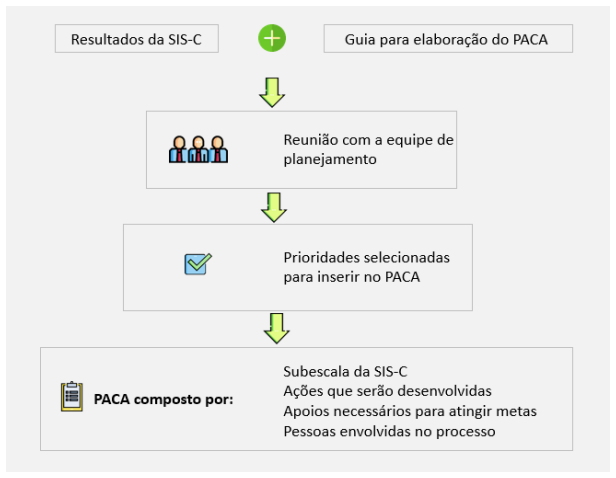

Partiu-se da verificação dos resultados da SIS-C respondido pela mãe e pela professora; a elaboração de um guia tendo como base o Guia para Planejamento Centrado na Pessoa com a Escala de Intensidade de Apoio para Adultos (Thompson et al., 2017) e o Manual do Usuário da Escala de Intensidade de Apoio para Crianças (Thompson et al., 2016); a realização de uma reunião com a mãe e com a equipe escolar de cada aluno, com 0 objetivo de elencar as prioridades de ações e apoios para os alunos; e o desenvolvimento do PACA de acordo com as prioridades elencadas pela equipe de planejamento.

\section{RESULTADOS E DISCUSSÃO}

Para este estudo serão apresentados os resultados e a discussão referente apenas ao participante Luizinho, sendo que essa escolha foi realizada de forma aleatória e é justificada pela extensão do material completo. Vale ressaltar que o foco deste estudo está no processo para a elaboração do PACA, sendo que os resultados da SIS$\mathrm{C}$, as prioridades elencadas serviram como embasamento para o planejamento do PACA.

\section{Luizinho}

A Figura 4 mostra que a mãe de Luizinho percebe que seu filho necessita de maior intensidade de apoio nas Atividades de Vida Comunitária, comparadas com as outras subescalas da SIS-C. Essas são atividades que exigem autonomia na mobilidade e, diante de seu diagnóstico (Paralisia Cerebral), Luizinho necessita de maior intensidade de apoio para deslocar-se, pois faz uso de cadeiras de roda.

A professora de Luizinho respondeu que 0 aluno necessita de maior apoio nas Atividades de Participação Escolar. As ações prioritárias elencadas pela equipe também estão expostas na Figura 4. 


\section{ESTRATÉGIAS NA ELABORAÇÃo dE PLANOS DE APOIO PARA ALUNOS COM DESENVOLVIMENTO ATíPICO}

Figura 4 - Atividades da SIS-C em que a mãe e a professora percebem que Luizinho necessita de maior intensidade de apoio, comparadas com as outras subescalas da SIS-C e as prioridades selecionadas pela equipe de planejamento



Fonte: Elaborado a partir de Thompson et al. (2016)

\section{Plano de Apoio Centrado no Aluno (PACA)}

Para cada aluno foi elaborado um Plano de apoio, baseado nas prioridades elencadas pela equipe de planejamento durante 0 encontro presencial. Com 0 intuito de complementar o plano, foram sugeridas atividades para desenvolver as prioridades propostas e inseridos materiais de apoio de acordo com as particularidades de cada aluno. Os QUADROS 1 e 2 apresentam algumas partes do PACA de Luizinho.

Quadro 1- PACA de Luizinho referente aos apoios para atividades em casa

\begin{tabular}{|c|c|c|c|}
\hline \multirow{2}{*}{$\begin{array}{c}\text { ATIVIDADES EM } \\
\text { CASA }\end{array}$} & AÇÕES & $\begin{array}{c}\text { QUAIS APOIOS } \\
\text { SÃO NECESSÁRIOS? }\end{array}$ & $\begin{array}{c}\text { QUEM IRÁ } \\
\text { AUXILIÁ-LO? }\end{array}$ \\
\hline & $\begin{array}{l}\text { - Incentivar o uso do } \\
\text { computador para } \\
\text { realizar pesquisas } \\
\text { escolares e procurar } \\
\text { assuntos que tenha } \\
\text { interesse. }\end{array}$ & $\begin{array}{l}\text { - Adaptar o computador ou } \\
\text { outro dispositivo eletrônico } \\
\text { para que ele tenha maior } \\
\text { autonomia no manuseio }{ }^{2} \text {. }\end{array}$ & $\begin{array}{l}\text { - Familiares; } \\
\text { - Terapeutas; } \\
\text { - Professor (a) da Sala de } \\
\text { Recursos Multifuncionais. }\end{array}$ \\
\hline \multicolumn{4}{|c|}{$\begin{array}{l}\text { *2. SUGESTÕES DE ESTRATÉGIAS: } \\
\text { - A mãe conversar com a terapeuta ocupacional e verificar a possibilidade de adaptar dispositivos eletrônicos para } \\
\text { que o aluno utilize em casa e na escola. } \\
\text { - Envolver a terapeuta ocupacional e a equipe pedagógica para traçar estratégias que envolvam a TECNOLOGLA } \\
\text { ASSISTIVA. Tecnologia Assistiva é o conjunto de recursos e serviços que contribuem para proporcionar ou } \\
\text { ampliar habilidades funcionais de pessoas com algum tipo de deficiência, incapacidade ou mobilidade reduzida, } \\
\text { podendo variar de uma simples bengala a um sistema computadorizado. No ambiente escolar, as tecnologias } \\
\text { assistivas ajudam a trazer mais autonomia e produtividade para os profissionais com deficiência fisica, auditiva ou } \\
\text { visual que necessitam de ajudas técnicas para realizar suas atividades diárias. }\end{array}$} \\
\hline
\end{tabular}


Quadro 2- PACA de Luizinho referente aos apoios para participação e aprendizagem escolar

\begin{tabular}{|c|c|c|c|}
\hline \multirow{6}{*}{$\begin{array}{c}\text { PARTICIPAÇÄO E } \\
\text { APRFNDIZAGEM } \\
\text { ESCOLAR }\end{array}$} & AÇÕES & $\begin{array}{l}\text { QUAIS APOIOS } \\
\text { SAO NECESSARIOS? }\end{array}$ & $\begin{array}{c}\text { QUEMIRA } \\
\text { AUXILIA-LO? }\end{array}$ \\
\hline & $\begin{array}{l}\text { - Incentivar o uso do } \\
\text { computador para realizar } \\
\text { pesquisas escolares e } \\
\text { procurar assumtos que } \\
\text { tenha interesse. }\end{array}$ & $\begin{array}{l}\text { - Adaptar o computador ou } \\
\text { outro dispositivo eletrônico } \\
\text { para que ele tenha maior } \\
\text { autonomia no manuseio. }\end{array}$ & $\begin{array}{l}\text { - Familiares } \\
\text { - Terapeutas } \\
\text { - Professor (a) da Sala de } \\
\text { Recursos } \\
\text { Multifuncionais. }\end{array}$ \\
\hline & \multirow[b]{2}{*}{ - Estimular a leitura } & Armentro 7 fonta dos & \\
\hline & & $\begin{array}{l}\text { Aumentar a fonte das } \\
\text { letras; } \\
\text { - Incentivar a leitura de } \\
\text { materiais que ele tem } \\
\text { interesse; } \\
\text { - Utilizar ferramentas } \\
\text { eletrônicas (computador ou } \\
\text { tablot) para realizar leituras. }\end{array}$ & $\begin{array}{l}\text { - Professores } \\
\text { - Familiares }\end{array}$ \\
\hline & \multirow[t]{2}{*}{$\begin{array}{l}\text { - Aprendizagem da } \\
\text { matemática }\end{array}$} & $\begin{array}{l}\text { - Utilizar materiais } \\
\text { concretos, como: } \\
\text { calculadora, palitos... } \\
\text { - Adequar o curriculo da } \\
\text { disciplina de matemática. } \\
\text { Ex.: diminuir a numeração. }\end{array}$ & - Equipe pedagógica \\
\hline & & $\begin{array}{l}\text { - Incentivar o uso da } \\
\text { matemática no dia a dia }{ }^{3} \text {. }\end{array}$ & - Familiares \\
\hline \multicolumn{4}{|c|}{$\begin{array}{l}\text { \#3. SUGESTÕES DE ATIVIDADES PARA REALIZAR NO CONTEXTO FAMILIAR: } \\
\text { - Envolvê-lo com o sistema monetário. Por exemplo: verificar quando precisa de troco, qual a soma de dois } \\
\text { produtog, se o dinheiro é suficiente para comprar algo; } \\
\text { - Incentivar o uso da calculadora para realizar cálculos necessärios para o dia a dia; }\end{array}$} \\
\hline
\end{tabular}

Compreender que todos os seres humanos precisam ser considerados como um sistema aberto, passível de ser significativamente modificado diante de diferentes estratégias (Feuerstein, Rand \& Feurstein, 2006), é um pensamento coerente com a mudança de entendimento sobre a deficiência, atualmente focado no funcionamento humano de acordo com as necessidades individuais de apoio (Buntinx, 2014).

Esses são princípios necessários para embasar estratégias na elaboração de planejamentos voltados para cada aluno. A partir desse cenário que envolve os apoios como princípios centrais para o funcionamento humano (Schalock et al., 2010), o presente estudo teve o privilégio de envolver famílias e equipes pedagógicas para desenvolver estratégias prioritárias para aprimorar o funcionamento adaptativo de cada aluno-alvo.

Os resultados da SIS-C foram essenciais para oferecer subsídios e seguir o Guia para a elaboração do PACA. Sendo assim, espera-se que no Brasil a escala também seja validada ${ }^{4}$, para servir como ferramenta para a construção de planos de apoios individuais, bem como para a alocação de recursos (Thompson et al., 2016).

Os planos individuais no Brasil ainda costumam focar nas informações básicas sobre o aluno, como: quanto tempo está na escola, aprendizagens que já foram consolidadas, dificuldades encontradas, metas, recursos e entre outros, para então estruturar um plano educacional individualizado, que busca respostas educativas às necessidades específicas do aluno no decorrer da aprendizagem (Glat, Vianna \& Redig, 2012). Na legislação federal não há dispositivos que garantem o PEl para o público alvo da educação especial matriculados nas escolas públicas regulares, para estes estudantes os serviços continuam centrados nas salas de recursos multifuncionais e centros de atendimento educacional especializado (Valadão \& Mendes, 2018). 


\section{ESTRATÉGIAS NA ELABORAÇÃo DE PLANOS DE APOIO PARA ALUNOS COM DESENVOLVIMENTO ATíPICO}

Indica-se pelo menos quatro encontros-com a equipe de planejamento, sendo necessário a participação da família, profissionais envolvidos com 0 aluno e, se adequado, o próprio aluno (Thompson et al., 2016, 2017). Para o presente estudo, as prioridades elencadas foram realizadas apenas em uma reunião, o que se mostrou insuficiente para completar todo 0 Guia. Espera-se que outras pesquisas sejam realizadas e que 0 material instrumental seja utilizado por outros autores, já que ainda é inexistente um Guia para a elaboração do Plano Centrado no aluno utilizando a SIS-C.

Por este estudo se caracterizar como uma pesquisa colaborativa, esta foi a oportunidade de cada membro da equipe contribuir com sua experiência profissional e vivencial com 0 aluno. Pode-se dizer que envolveu uma etapa de consultoria colaborativa, definida por Bello, Machado e Almeida (2012) como uma proposta de atuação conjunta com o intuito de mutuamente resolverem os problemas através do compartilhamento de saberes e responsabilidades.-

É necessário ressaltar que o PACA foi elaborado como uma sugestão de ferramenta de apoio para aprimorar 0 funcionamento adaptativo dos alunos. Thompson et al. $(2016,2017)$ sugerem que ocorra constantemente 0 monitoramento e avaliação da eficácia dos apoios apontados como necessários.

Para Thompson et al. (2016), o trabalho de identificar, organizar e implementar apoios é um processo e não tem um fim. Assim como os alunos mudam, as atividades devem mudar; os contextos devem se transformar e as equipes de planejamento devem se propor a adquirir mais conhecimento e experiência.

\section{CONCLUSÕES}

Utilizar estratégias que envolvam uma análise prévia sobre os apoios necessários para que cada aluno se desenvolva com maior autonomia e qualidade de vida é necessário para o desenvolvimento de planos individuais (Schalock et al., 2010; Herps, Buntinx \& Curfs, 2013). A partir dos resultados foi possível alcançar o objetivo traçado para este estudo, especificando as estratégias utilizadas para chegar à elaboração do PACA. Também se atingiu os objetivos específicos, dentre os quais cita-se a descrição das prioridades elencadas pela equipe de planejamento e, como elemento final, a elaboração do PACA.

Vale ressaltar que as estratégias escolhidas pela pesquisadora foram embasadas em pesquisas, publicações (Pletsch \& Glat, 2012; Herps, Buntinx \& Curfs, 2013; Robertson et al., 2017) e manuais de colaboradores da AAIDD (Schalock et al., 2010; Thompson et al., 2016, 2017). Subsidiar as estratégias com uma avaliação prévia (SIS-C) e utilizar um guia para direcionar a reunião foram apoios essenciais para a elaboração do PACA.

Conforme mencionado na discussão, foi realizada apenas uma reunião com a equipe de planejamento, priorizando as principais ações e não preenchendo todo o Guia. Isso ocorreu pela dificuldade que a pesquisadora encontrou de reunir a equipe em outros encontros, mas essa limitação não impediu a elaboração do plano, logo com mais encontros outras ações poderiam ter sido elencadas. Entende-se que outras pesquisas também podem contribuir, avaliando a intensidade de apoio para comportamentos adaptativos antes e após a intervenção das atividades propostas no PACA do aluno. Essa avaliação pode ser realizada com a aplicação da SIS-C.

Considera-se as seguintes contribuições da presente pesquisa para futuros estudos que pretendem desenvolver planos individuais: a utilização de instrumentos ainda pouco explorados no Brasil (SIS-C e PACA) e 0 reforço da necessidade de encontros presenciais envolvendo a família, a professora e outros membros da equipe escolar a fim de compartilhar conquistas apresentadas pelo aluno e a soma de experiências.

Pode-se dizer que o produto final é o PACA, mas todo o processo de construção desse Plano é a maior contribuição desta pesquisa para futuros profissionais. Espera-se que outros pesquisadores possam usufruir das estratégias descritas neste estudo, mas que também ousem construir outras maneiras de elaborar planos individuais que contribuam para o bem-estar, a autonomia e a participação plena dos alunos com desenvolvimento atípico. 


\section{REFERÊNCIAS BIBLIOGRÁFICAS}

AMERICAN PSYCHIATRIC ASSOCIATION [APA]. Manual Diagnóstico e Estatístico de Transtornos Mentais. Tradução de: NASCIMENTO, M. I. C.; MACHADO, P. H.; GARCEZ, R. M.; PIZZATO, R.; ROSA, S. M. M. 5. ed. Porto Alegre: Artmed, 2014. Título original: Diagnostic and Statistical Manual of Mental Disorders.

BARIL, N. Avaliação de intensidade de apoio de comportamentos adaptativos e elaboração de plano centrado no aluno com desenvolvimento atípico [dissertação de mestrado - Setor de Educação]: Universidade Federal do Paraná. Disponível em: < https://acervodigital.ufpr.br/bitstream/handle/1884/63808/R\%20-\%20D\%20-\%20NATHALIE\%20BARIL.pdf?sequence=1\&isAllowed=y >. Acesso em: 25 maio de 2020.

BELLO, S., MACHADO, A. C. \& ALMEIDA, M. A. Parceria colaborativa entre fonoaudiólogo e professor: análise dos diários reflexivos. Rev. Psicopedag, [online], v. 29, n. 88, s/p, 2012.

BRASIL. Decreto n. 7.611, de 17 de novembro de 2011. Dispõe sobre 0 atendimento educacional especializado. Brasília: Presidência da República/Casa Civil/Subchefia para Assuntos Jurídicos, 2011.

BRASIL. Lei $\mathbf{n}^{\mathbf{0}} \mathbf{1 3 . 1 4 6}$, de $\mathbf{6}$ de julho de 2015. Institui a lei brasileira de inclusão da pessoa com deficiência (estatuto da pessoa com deficiência). Diário Oficial da União, Brasília, DF, 07 jul. 2015. p. 2.

BRASIL, Ministério da Educação. Plano Nacional de Educação. 0 PNE 2011-2020: metas e estratégias. Brasília: MEC, 2010.

BUNTINX, W. Understanding disability: a strenghts-based approach. In: WEHMEYER, Michael. The Oxford handbook of Positive Psychology and Disability. Nova York: Oxford University Press, 2014.DESSEN, Maria Auxiliadora.; POLONIA, Ana da Costa. A família e a escola como contextos de desenvolvimento humano. Paidéia, Ribeirão Preto, v. 17, n. 36, p. 21-32, 2007.

FELCE, D. Can Person-Centred Planning fulfil a strategic planning role? Comments on Mansell \& Beadle-Brown. Journal of Applied Research in Intellectual Disabilities, London, v. 17, p. 27-30, 2004.

FEUERSTEIN, R., RAND, Y. \& FEUERSTEIN, R. You love me! Don t accept as I am. Jesusalem: ICELP Publications, 2006.

GLAT, R., VIANNA, M. M. \& REDIG, A. G.. Plano Educacional Individualizado: uma estratégia a ser construída no processo de formação docente. Revista Universidade Rural, Rio de Janeiro, v. 34, p. 108-129, 2012.

HERPS, M., BUNTINX, W. \& CURFS, L. Individual support planning: perceptions and expectations of people with intellectual disabilities in the Netherlands. Journal of Intellectual Disability Research, London, v. 57, n. 11, p. 1027-1036, 2013. doi: 10.1111/j.1365-2788.2012.01598.x

HERPS, M. A.,et al. Individual supports plans of people with intellectual disabilities in residential services: content analysis of goals and resources in relation to client characteristics. J Intellect Disabil Res., London, v. 60 , p. 3, p.254-262, 2016.

MELLO, A. de F. G. \& HOSTINS, R. C. L. Construção mediada e colaborativa de instrumentos de avaliação da aprendizagem na escola inclusiva. Revista Educação Especial, Santa Maria, v. 31, n. 63, p. 1025-1038, out./dez. 2018. doi: 10.5902/1984686X33101

PARANÁ. Conselho Estadual de Educação. Deliberação n. 02/2016. Dispõe sobre as normas para a modalidade Educação Especial no Sistema Estadual de Ensino do Paraná. Diário Oficial Executivo, n. 9788, 23 set. 2016. p. 12.

PLETSCH, M. D. \& GLAT, R. A escolarização de alunos com deficiência intelectual: uma análise da aplicação do Plano de Desenvolvimento Educacional Individualizado. Linhas Críticas, Brasília, v. 18, n. 35, p. 193-208, 2012. 


\section{ESTRATÉGIAS NA ELABORAÇÃo dE PLANOS DE APOIO PARA ALUNOS COM DESENVOLVIMENTO ATÍPICO}

PLETSCH, M., et al. Plano de Desenvolvimento Psicoeducacional Individualizado (PDPI): estratégia para favorecer o processo de ensino e aprendizagem de alunos com deficiência intelectual. In: CONGRESSO BRASILEIRO DE EDUCAÇÃO ESPECIAL, IV, 2010; ENCONTRO NACIONAL DE PESQUISADORES EM EDUCAÇÃO ESPECIAL, IV, 2010, São Carlos. Anais... Programa de Pós-Graduação em Educação Especial; Associação Brasileira de Pesquisadores em Educação Especial (ABPEE), 2010. 1-10.

ROBERTSON, J., et al. Person-centred planning: factors associated with successful outcomes for people with intellectual disabilities. J Intellect Disabil Res., London, v. 51, p. 3, p. 232-243, mar./2017.

SCHALOCK, R., et al. Intellectual Disability: definition, classification, and systems of supports. 11. ed. Washignton, DC: American Association on Intellectual and Developmental Disabilities, 2010.

TANNUS-VALADAO, G. \& MENDES, E. G. Inclusão escolar e o planejamento educacional individualizado: estudo comparativo sobre práticas de planejamento em diferentes países. Rev. Bras. Educ., Rio de Janeiro, v. 23, e230076, 2018. doi: 10.1590/s1413-24782018230076.

THOMPSON, J., et al. SIS-A: Person-centered Planning with the Supports Intensity Scale- Adult Version. A Guide for planning teams. Washington, DC: American Association on Intellectual and Developmental Disabilities, 2017.

THOMPSON, J. \& VIRIYANGKA. Supports and supports needs. In: WEHMEYER, Michael. The Oxford Handbook of Positive Psychology and Disability. New York: Oxford Library of Psychology, 2014. p. 317-337.

THOMPSON., et al. Supports Intensity Scale- Children s Version. User s Manual. Washington, DC: American Association on Intellectual and Developmental Disabilities, 2016.

VAN LOON, J. Planes individuales de apoyo: mejora de los resultados personales. Individual Support Plans: enhancing personal outcomes. BC Middelburg, The Netherlands: Fundación Arduin y Departamento de Educación Especial de la Universidad de Gante, 2015. doi: 10.14201/scer020154612540

\section{NOTAS}

10 termo TGD aqui empregado faz referência ao utilizado na resolução citada (BRASIL, 2011), porém, sabese que o utilizado usualmente é o citado pelo DSM-V (APA, 2014), ou seja, Transtorno do Espectro Autista.

${ }^{2}$ Copyright@ 2015 American Association on Intellectual and Developmental Disabilities. All rights reserved..

${ }^{3}$ As imagens foram escolhidas aleatoriamente e são meramente ilustrativas, não correspondendo a quaisquer características físicas dos alunos.

${ }^{4}$ A escala está sendo validada através do projeto "Adaptação e validação da Escala de intensidade de apoio para crianças e adolescentes (SIS-C) com deficiência intelectual no Brasil", uma parceria entre a UFPR, a UFSM, e a Federação Nacional das APAE s e com permissão para pesquisa da American Association on Intellectual and Developmental Disabilities ("AAIDD"). 